\title{
PHYSICAL PROPERTIES OF SUBCRITICAL ACCRETION DISKS
}

\author{
O. M. HEINRICH \\ Institut f. Theoretische Astrophysik \\ Tiergartenstr. 15, 69121 Heidelberg
}

Despite of the great interest in accretion disks around supermassive black holes there are still numerous controversies about their structure and their physical properties. Therefore we have calculated the structure of such disks for a wide range of parameters. For the viscosity we use a standard $\alpha$ description with the main component of the stress tensor $t_{r \phi}$ proportional to the gas pressure. The disk structure depends sensitively on material properties such as opacities and specific heats. In our calculations we have used an equation of state and mean opacities taking into account a list of the most important ionization processes as well as the radiation contributions to thermodynamic quantities.

The most interesting features of our models can be summarized as follows: - Accretion disks around supermassive black holes are optically very thick due to a rather high surface density $\left(\Sigma \approx 10^{5} \ldots 10^{7} \mathrm{~g} / \mathrm{cm}^{2}\right)$. This means that the radial inflow velocity is almost everywhere (except a very narrow boundary layer near the inner edge) very small compared with the velocity of sound and thus advection effects are negligible. Furthermore, the timescale for the viscous evolution is very long.

- Since these disks are very massive, selfgravity becomes important for $r \approx 10^{3} r_{g}$ and the disk is subject to a gravitational instability. This might indicate that the outer part of the disk is fragmented and a region of intensive star formation. The wind from such a surrounding stellar torus may considerably contribute to the feeding of the inner accretion region.

- In the inner part of the disk radiation pressure dominates over gas pressure. Nevertheless the dominant energy transport mechanism is not radiative transfer but turbulent heat transport.

Heinrich, O.M. (1994) Astron. Astrophys. 286, pp. 338-343

Falcke, H. and Heinrich, O. M. (1994) Astron. Astrophys. 292, pp. 430-438 